\title{
Luteolin suppresses development of medroxyprogesterone acetate-accelerated 7,12-dimethylbenz(a)anthracene-induced mammary tumors in Sprague-Dawley rats
}

\author{
MATTHEW T. COOK ${ }^{1}$, BENFORD MAFUVADZE ${ }^{1}$, CYNTHIA BESCH-WILLIFORD $^{2}$, \\ MARK R. ELLERSIECK ${ }^{3}$, SANDY GOYETTE ${ }^{1}$ and SALMAN M. HYDER ${ }^{1}$ \\ ${ }^{1}$ Department of Biomedical Sciences and Dalton Cardiovascular Research Center, University of Missouri, Columbia, \\ MO 65211; ${ }^{2}$ IDEXX BioResearch, Columbia, MO 65202; ${ }^{3}$ Agriculture Experiment Station, \\ University of Missouri, Columbia, MO 65211, USA
}

Received September 28, 2015; Accepted October 23, 2015

DOI: 10.3892/or.2015.4431

\begin{abstract}
Postmenopausal women undergoing hormonereplacement therapy containing both progestins and estrogens are at an increased risk of developing breast cancer compared with women taking estrogen alone. We recently demonstrated that medroxyprogesterone acetate, a progestin commonly used for hormone-replacement therapy, accelerates development of mammary carcinogenesis in 7,12-dimethylbenz(a) anthracene-treated Sprague-Dawley rats. Synthetic antiprogestins used to block the deleterious effects of progestins, are themselves associated with toxic side-effects. In order to circumvent this, we used the aforementioned model to identify less toxic natural compounds that may prevent the development of progestin-accelerated tumors. Luteolin, a naturally-occurring flavonoid commonly found in fruits and vegetables, has previously been shown to possess anticancer properties. In our studies, both low (1 mg/kg) and high $(25 \mathrm{mg} / \mathrm{kg})$ doses of luteolin significantly suppressed progestin-dependent increases in tumor incidence, while increasing tumor latency and reducing the occurrence of large $\left(>300 \mathrm{~mm}^{3}\right)$ mammary tumors. However, an intermediate dose of luteolin $(10 \mathrm{mg} / \mathrm{kg})$, while suppressing the development of large tumors, did not affect either tumor incidence or latency. Immunohistochemical analysis of tumor tissues revealed that all concentrations of luteolin $(1,10$, and $25 \mathrm{mg} / \mathrm{kg})$ significantly reduced levels of VEGF within tumors. The suppressive effects of luteolin on tumor incidence and volume, together with its ability to reduce VEGF and blood vessels, persisted even after treatment was terminated. This suggests that luteolin possesses anti-angio-
\end{abstract}

Correspondence to: Dr Salman M. Hyder, Dalton Cardiovascular Research Center, University of Missouri, 134 Research Park Drive, Columbia, MO 65211, USA

E-mail: hyders@missouri.edu

Key words: luteolin, breast cancer, medroxyprogesterone acetate, chemoprevention genic properties which could mechanistically explain its capacity to control tumor progression. Thus luteolin may be a valuable, non-toxic, naturally-occurring anticancer compound which may potentially be used to combat progestin-accelerated mammary tumors.

\section{Introduction}

Breast cancer is the second most commonly diagnosed type of cancer, and the leading cause of cancer-related death in American women. In the United States, 240,000 mostly postmenopausal women are diagnosed with the disease every year (1). A number of recent independent clinical trials and studies have shown that postmenopausal women undergoing combined estrogen and progestin hormone-replacement therapy (HRT) have an increased risk of developing metastatic breast cancer compared with women taking only estrogen (2-5). Combination HRT is commonly prescribed to women with an intact uterus to alleviate postmenopausal symptoms. The progestin component is added to minimize the risk of endometrial hyperplasia, which may precede endometrial cancer $(6,7)$. Recent laboratory studies have shown that progestin stimulates proliferation of normal and neoplastic breast cancer cells $(8,9)$, correlating with clinical findings for combination HRT use.

Studies designed to elucidate the mechanism(s) underlying the increased incidence of breast cancer associated with combination HRT have shown that progestins induce the potent angiogenic factor VEGF, in human breast cancer cells (10-12). Furthermore, such studies have demonstrated that both natural and synthetic progestins increase the synthesis and secretion of VEGF in breast cancer cells that express mutant p53 tumor-suppressor protein, but not wild-type p53 $(12,13)$. Locally produced VEGF acts in a paracrine manner to stimulate both endothelial and tumor epithelial cells; the latter cells are also stimulated by VEGF in an autocrine fashion (14). In addition, progestins such as medroxyprogesterone acetate (MPA), a commonly prescribed component of HRT, have been implicated in reactivating breast cancer stem cell subpopulations in hormone-responsive cell lines $(12,15)$. Progestins are also believed to act in concert with RANKL to increase cellular 
proliferation $(9,16)$, and increase tumor vasculature $(12,17)$, thereby providing an enriched environment for tumor growth and metastasis.

Previous studies in our laboratory have shown that progestins drive 7,12-dimethylbenz(a)anthracene (DMBA)-induced hormone-dependent mammary tumors in Sprague-Dawley rats (17-19). We have exploited this finding to establish an in vivo model for testing progestin antagonists in an inclusive microenvironment. Although the exact mechanism behind progestin-accelerated tumor growth in the DMBA model is not fully understood, evidence suggests that progestin-induced VEGF production resulting in increased angiogenesis is likely responsible (17-19). Previous studies have reported that antiprogestins (both synthetic, such as RU-486, and naturallyoccurring compounds, such as apigenin) block the incidence and growth of mammary tumors in the progestin-accelerated DMBA-induced model (17,19-21). These studies suggest that progestin-accelerated DMBA-induced mammary tumor incidence is largely influenced by the inherent ability of progestins to increase production of VEGF, resulting in decreased latency and increased tumor incidence, burden, and multiplicity. For this reason, the model provides an excellent means of studying hormone-dependent breast cancer and is particularly suitable for identifying naturally-occurring, non-toxic antagonists of progestin-induced VEGF.

Luteolin [2-(3,4-dihydroxyphenyl)-5,7-dihydroxy4H-1-benzopyran-4-one] (LU) is a low molecular weight, naturally-occurring flavonoid commonly found in fruits and vegetables. A number of studies show that LU possesses a myriad of anticancer functions and that it suppresses tumor development in several types of human cancer more effectively than other flavonoids (22-24). Studies show that LU inhibits VEGF production and that it has antiprogestin capabilities (25-29), demonstrating its potential effectiveness against hormone-responsive cancers. Collectively, these findings suggest that LU has important chemopreventive properties. Herein, we provide evidence supporting the ability of LU to substantially inhibit MPA-accelerated tumor latency, incidence and growth in the DMBA-induced mammary tumor model. We also show that LU possibly arrests the growth of mammary tumors by suppressing VEGF production and angiogenesis, vital components of breast tumor formation and development.

\section{Materials and methods}

Animals. All surgical and experimental procedures were approved by the University of Missouri-Columbia Institutional Animal Care and Use Committee (IACUC). Intact adult female Sprague-Dawley rats (45-55-day old) were purchased from Harlan Breeders (Indianapolis, IN, USA) and maintained under 12-h light/dark cycles with ad libitum access to food (LabDiet 5008; LabDiet, St. Louis, MO, USA) and water in accordance with guidelines established by the Association for Assessment and Accreditation of Laboratory Animal Care International (AAALAC).

Luteolin. LU was purchased from Indofine Chemical Company (cat no. L-101; Hillsborough, NJ, USA) and dissolved in sterile filtered dimethyl sulfoxide (DMSO, cat no. D2650;
Sigma-Aldrich, St. Louis, MO, USA). Solutions of LU were prepared weekly, aliquoted for daily use, and stored at $-20^{\circ} \mathrm{C}$ until use.

Experimental design. We modified the protocol of DMBA-induced mammary tumor formation described previously $(17,19)$ (summarized in Fig. 1). Animals were given a $1 \mathrm{ml}$ bolus of $10 \mathrm{mg}$ of DMBA (cat no. D3254; Sigma-Aldrich) dissolved in peanut oil by oral gavage (day 0). Three weeks post-DMBA administration (day 21), animals were divided into 5 treatment groups ( $\mathrm{n}=10-12$ animals/group). Animals in the control group and those given only MPA were administered DMSO by intraperitoneal injection. The animals given $\mathrm{LU}(1,10$, or $25 \mathrm{mg} / \mathrm{kg})$ received injections of the flavonoid in DMSO every $24 \mathrm{~h}$ for 10 days, followed by another 8 injections at 48 -h intervals. LU doses were selected based on previously reported in vivo studies (30-32). Four weeks postDMBA administration (day 28), $25 \mathrm{mg}$ 60-day release MPA or placebo pellets (cat no. P-161; Innovative Research of America, Sarasota, FL, USA) were implanted subcutaneously on the dorsal part of the neck. Animals were weighed twice a week and, starting on day 29 , palpated every other day to detect tumor latency and incidence. On day 59, all animals were sacrificed and tumor number and volume $\left(1 / 2 \mathrm{~L} \mathrm{x} \mathrm{W}^{2}\right)(33,34)$ determined. Tumors and contralateral inguinal mammary gland tissue devoid of tumors were retained for analysis.

Histology and immunohistochemical analysis. Immunohistochemical staining of mammary and tumor tissue was performed following previously described procedures $(17,19)$. The following polyclonal antibodies were used: antiVEGF antibody (1:100 dilution, cat no. SC-152; Santa Cruz Biotechnology Dallas, TX, USA); and anti-Ki67 antigen antibody (1:400 dilution, cat no. RB1510-P; Thermo Scientific Waltham, MA, USA). Cell death immunohistochemistry was determined using a Roche (Basel, Switzerland) terminal deoxynucleotidyl transferase-mediated dUTP nick end labeling (TUNEL) detection kit. Histological samples were analyzed and quantified using Fovea Pro 3.0 (Reindeer Graphics) and ImageJ. Images were captured at $\mathrm{x} 20$ and $\times 40$ magnification and threshold intensity was adjusted for measurement in pixels. Tumors and representative contralateral inguinal mammary gland tissues were excised from animals in each treatment group, fixed in formaldehyde, and embedded in paraffin for immunohistochemistry. One section from each individual tumor and mammary gland was placed on the corresponding slide for each of the immunohistochemical stains and 4 random fields captured from every section to minimize errors due to differences in cellularity. All the mammary tumors were collected and assessed for IHC biomarkers. The availability of tumor sections was dependent upon tumor occurrence (while 6-9 tumors developed in control, MPA and MPA $+10 \mathrm{mg}$ / $\mathrm{kg} \mathrm{LU}$ groups, only 2 and 3 animals developed tumors in the 1 and $25 \mathrm{mg} / \mathrm{kg} \mathrm{LU}$-treated animal group, respectively). Regions of staining within tumors and areas of mammary hyperplasia in contralateral inguinal mammary gland tissue were recorded. For analysis of mammary gland tissue, only four glands were used in each group. Fovea Pro 3.0 was used to quantitate the percent area of VEGF, Ki67 and TUNEL staining in tumor tissue using the color threshold feature 


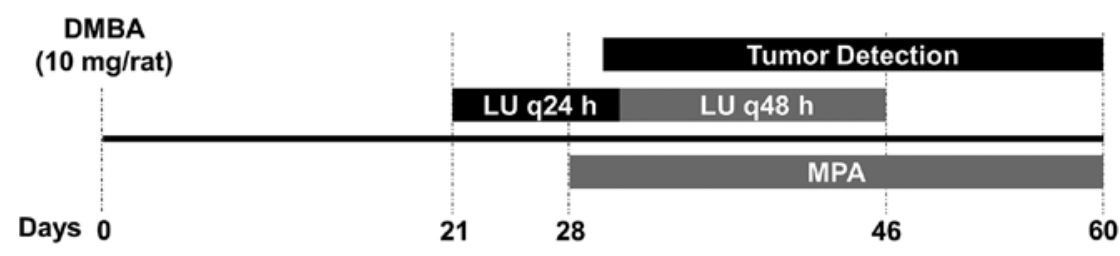

Figure 1. Luteolin treatment protocol. Sprague-Dawley rats were given $10 \mathrm{mg}$ DMBA orally and subsequently implanted with a $25 \mathrm{mg} 60$-day release MPA (or placebo) pellet on day 28 as described in Materials and methods. Luteolin (LU; 1, 10, or $25 \mathrm{mg} / \mathrm{kg}$ ) or vehicle (DMSO) was injected every $24 \mathrm{~h}$ (q24 h) for 10 days beginning on day 21, and then every $48 \mathrm{~h}(\mathrm{q} 48 \mathrm{~h}$ ) until day 46 . Animals were palpated for tumors every other day beginning on day 29 and continuing until day 59.

A

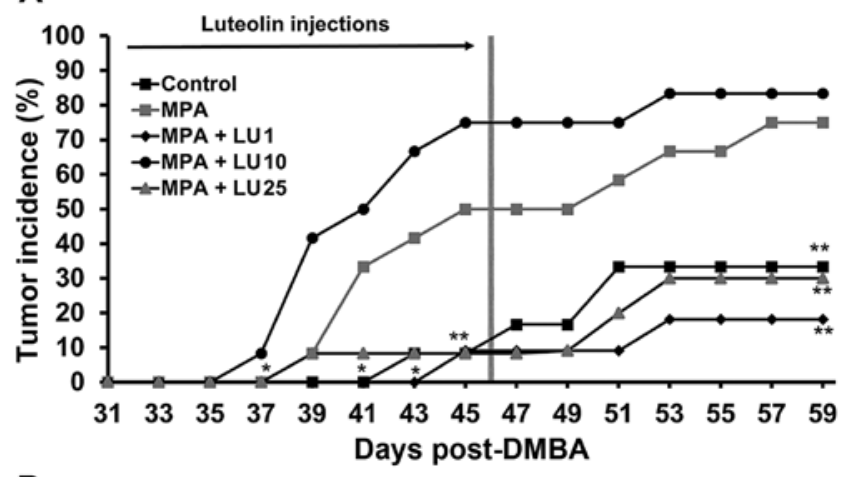

B

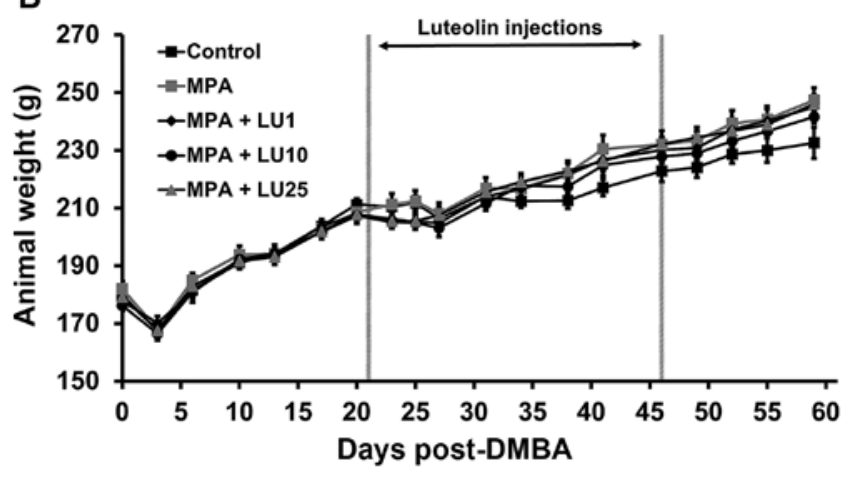

Figure 2. Luteolin prevents development of DMBA-induced MPA accelerated tumors. (A) Effects of LU on tumor latency and tumor incidence. Curves are compared for significance. ${ }^{*} \mathrm{P}<0.05$ for MPA +1 and $25 \mathrm{mg} / \mathrm{kg}$ LU groups and the control group compared with tumor latency in the MPA-treated group; ${ }^{* *} \mathrm{P}<0.05$ compared with tumor incidence in the MPA-treated group at day 45 and 59. Asterisks are placed above thge data points. Data represent the percent of animals with tumors in each treatment group at each time-point ( $\mathrm{n}=10-12$ animals/group). Control animals were given DMBA, implanted with a placebo pellet, and subjected to vehicle injections as per the protocol described in Fig. 1. MPA animals were treated identically to control animals, except that they were implanted with an MPA pellet rather than a placebo pellet on day 28. (B) Effects of LU on animal weights in the treatment groups throughout the study. Data represent the mean \pm SEM.

in ImageJ. This facilitated precise discrimination between positive/negative cells and background. CD31, a blood vessel marker, was used to quantitate blood vessels in excised tumor tissue. Three CD31-labeled x10 sections were taken from each tumor to minimize intratumoral variation, as previously described (12). The total number of vessels were counted in each section and then averaged per corresponding tumor. Data was then reported as means per treatment group with each group having an $\mathrm{n} \geq 3$; except for the group given $25 \mathrm{mg} / \mathrm{kg} \mathrm{LU}$, which contained 1 tumor.
Statistical analysis. Tumor latency was analyzed using the LIFETEST procedure in SAS software (9.4) to determine differences in time-to-event. Pairwise comparisons between groups were made using the Wilcoxon log-rank test in which the time-to-event represents time to appearance of the first tumor in each animal. Tumor-free animals were censored upon death or termination of the study (day 59). All other tumor burdened animals, regardless of survival, were uncensored. Tumor incidence and number of tumors per category (up to $300 \mathrm{~mm}^{3}$ or $>300 \mathrm{~mm}^{3}$ ) were compared pairwise using the general linear model (GENMOD) procedure in SAS software to determine the differences in least squared means among groups (logit link function and a binomial distribution). A logit link with a distribution binomial $p$ cannot be equal to $0[\operatorname{logit}=\ln (\mathrm{p} / 1-\mathrm{p})]$, thus the $\log$ of 0 is undefined. In the group treated with MPA $+25 \mathrm{mg} / \mathrm{kg}$ LU, there were 0 tumors formed in $>300 \mathrm{~mm}^{3}$ group, therefore a ' 1 ' was added to this group for statistical analysis (i.e., 1 tumor in 10 animals, instead of 0 in 10 animals). No adjustment was made for multiple comparisons in the LIFETEST or GENMOD procedures. Immunohistochemical data were analyzed using an ANOVA followed by an all pairwise multiple comparison test (Student-Newman-Keuls test) in SigmaPlot 12.5. IHC analysis of VEGF in the groups given MPA and MPA + LU $25 \mathrm{mg} / \mathrm{kg}$ was by t-test in SigmaPlot 12.5. In the group given $25 \mathrm{mg} / \mathrm{kg}$ LU (tumor tissue was $\mathrm{n}=1$ ), analysis of VEGF IHC by the Student-Newman-Keuls multi-range test met the critical value between MPA and MPA $+25 \mathrm{mg} / \mathrm{kg}$ LU due to the large difference between treatment groups, resulting in statistical significance. This test assumes that MPA $+25 \mathrm{mg} / \mathrm{kg} \mathrm{LU}$ is a true representation of the mean (\% area). Similar statistical significance was not reached with the MPA $+25 \mathrm{mg} / \mathrm{kg} \mathrm{LU}$ groups for other markers analyzed by IHC. For all comparisons, $\mathrm{P} \leq 0.05$ was regarded as statistically significant.

\section{Results}

Luteolin suppresses development of progestin-accelerated $D M B A$-induced mammary tumors. Using our well-established model of DMBA-induced mammary tumors (17-19), we examined the potential of LU to prevent MPA-driven tumor development. Three weeks after DMBA administration and 1 week prior to implantation of the MPA pellet (day 21), various doses of LU were administered to determine its ability to impede MPA-dependent tumor development by preventing the progression of neoplastic lesions to frank tumors. MPA reduced tumor latency in DMBA-treated rats compared with controls (DMBA-treated rats implanted with placebo pellets) (Fig. 2A; $\mathrm{P}<0.05$ ). Interestingly, the latency 


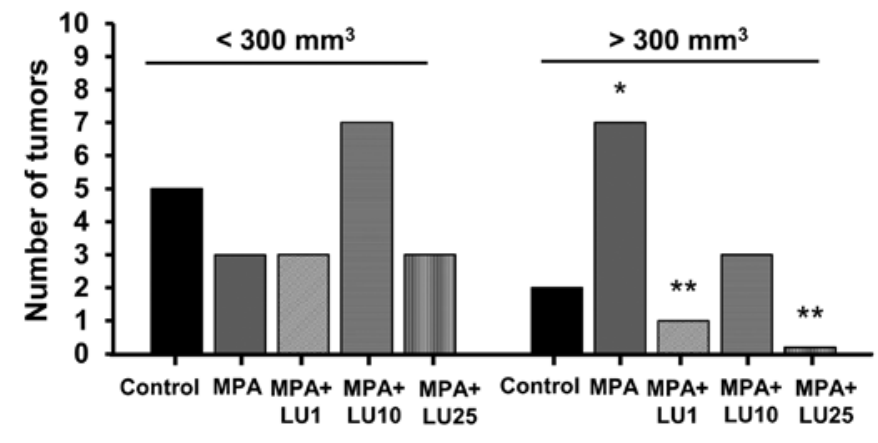

Figure 3. Luteolin suppresses MPA-driven growth of DMBA-induced mammary tumors. Evaluation of tumor size in different groups at the end of the LU treatment protocol. Tumor volumes were calculated, and the total number of tumors $<300$ and $>300 \mathrm{~mm}^{3}$ in each treatment group determined ${ }^{*} \mathrm{P}<0.05$ compared with the $>300 \mathrm{~mm}^{3}$ control group; ${ }^{* *} \mathrm{P}<0.05$ compared with the $>300 \mathrm{~mm}^{3}$ MPA-treated group. Please note that there were no tumors $>300 \mathrm{~mm}^{3}$ in the MPA + LU25 group.

curve for the $10 \mathrm{mg} / \mathrm{kg}$ LU group was similar to that of the MPA group, with no significant difference between the two profiles. In contrast, in those animals given MPA + either 1 or $25 \mathrm{mg} / \mathrm{kg} \mathrm{LU}$, time-to-event data (latency) increased significantly (LIFETEST; ${ }^{*} \mathrm{P}<0.05$ ) compared with animals given MPA alone.

At termination of LU treatment (day 46), tumor incidence increased in animals treated with MPA alone and $\mathrm{MPA}+10 \mathrm{mg} / \mathrm{kg}$ LU compared with controls and those administered 1 or $25 \mathrm{mg} / \mathrm{kg} \mathrm{LU}+\mathrm{MPA}(\mathrm{P}<0.05)$ (Fig. 2A). Following cessation of LU treatment, tumor incidence in animals receiving the flavonoid at a dose of 1 or $25 \mathrm{mg} / \mathrm{kg}$ remained relatively low until the end of the experiment on day 59. As a result, tumor incidence at day 59 in groups given 1 or $25 \mathrm{mg} / \mathrm{kg}$ LU and controls was significantly reduced compared with that in animals treated with MPA alone (Fig. 2A). Interestingly, administration of $10 \mathrm{mg} / \mathrm{kg} \mathrm{LU}$ appeared to have little or no inhibitory effect on MPA-driven tumor incidence (Fig. 2A). Animal weights were not significantly affected by LU at even the highest dose used $(25 \mathrm{mg} / \mathrm{kg})$ throughout these studies (Fig. 2B), indicating that the flavonoid had little or no toxicity.

Even though more than one tumor developed in a few animals (two animals in control, two in MPA, one in $\mathrm{MPA}+\mathrm{LU} 1$, one in MPA + LU10, and 0 in MPA + LU25 groups) there was no overall significant difference in tumor multiplicity between groups. The majority of tumors formed in this animal model were ductal carcinomas with cribriform, papillary or a combination of cribriform and papillary patterns. Ductal carcinomas were also the predominant type of neoplasm detected in LU-treated rats and there was no observable trend for a particular classification of neoplasm as a response to the different treatments.

Luteolin suppresses progestin-driven mammary tumor growth. Due to the biological variance in the volume of tumors within and among animal treatment groups (data not shown), they were divided into two size groups, separating them into small and large tumors (small, up to $300 \mathrm{~mm}^{3}$; large $>300 \mathrm{~mm}^{3}$ ).

No statistical differences were observed among the various treatment groups with respect to the numbers of small tumors $\left(<300 \mathrm{~mm}^{3}\right)$ occurring in experimental animals (Fig. 3), though more small tumors developed in the group given $\mathrm{MPA}+10 \mathrm{mg} / \mathrm{kg} \mathrm{LU}$, reflecting a higher incidence of tumors in this group compared with other LU-treated groups (Fig. 2A). However, the number of large $\left(>300 \mathrm{~mm}^{3}\right)$ tumors arising in animals receiving only MPA, was significantly higher than in the control group (Fig. 3). Administration of 1 or $25 \mathrm{mg} / \mathrm{kg} \mathrm{LU}$ significantly reduced the number of large tumors compared with the number observed in the MPA-treated group (Fig. 3), suggesting that LU interfered with MPA-driven tumor volume increases. Interestingly, although by day 59 no difference was observed in tumor incidence between animals given MPA alone and those administered MPA $+10 \mathrm{mg} / \mathrm{kg} \mathrm{LU}$ (Fig. 2A), more of the tumors in the latter group were small $\left(<300 \mathrm{~mm}^{3}\right)$. This finding suggests that a dose of $10 \mathrm{mg} / \mathrm{kg} \mathrm{LU}$, while not affecting tumor incidence, suppresses MPA-driven tumor growth and prevents the development of small tumors into larger ones.

Luteolin promotes mammary tumor regression. Our initial results demonstrated that doses of 1 or $25 \mathrm{mg} / \mathrm{kg}$ LU most effectively suppressed progestin-dependent increases in tumor incidence and growth. In the $1 \mathrm{mg} / \mathrm{kg}$ LU treatment group, a total of only 4 tumors were detected (in 2 of 11 animals), while just 3 tumors were observed in the group given $25 \mathrm{mg} / \mathrm{kg} \mathrm{LU}$ [in 3 of 10 animals (Fig. 2A)].

In the $25 \mathrm{mg} / \mathrm{kg}$ group, only 2 of the 3 tumors were present during the last week of LU treatment. These tumors were initially palpated on days 39 and 51, while the third tumor was first palpated on day 53. Tumors detected on days 51 and 53 developed well after termination of LU treatment. The tumor detected on day 53 contained a hypercellular stromal compartment surrounded by nests of cribriform, hyperplastic glandular tissue (Fig. 4). The first tumor which arose on day 39 during LU treatment had decreased in size by the time it was excised and examined on day 59 (Fig. 4). This mass was composed of tightly-packed tubular structures with empty lumens and lined with a flattened epithelium. The cause of this change is not known, but has features suggestive of epithelial atrophy. The tumor detected on day 51 was too small and not collectable at the end of the experiment. Consequently, the tumor that emerged on day 53 (Fig. 4) was used alone for all subsequent immunohistochemical analysis of tissues representing the $25 \mathrm{mg} / \mathrm{kg} \mathrm{LU}$ treatment group.

Luteolin reduces expression of VEGF and CD-31, markers of angiogenesis, in mammary tumor tissue. In previous studies, we showed that continuous production of VEGF by breast cancer cells is a vital component of MPA-dependent angiogenesis and subsequent tumor development $(12,17,19)$. In the current studies, we postulated that LU would reduce progestin-accelerated tumor growth by suppressing MPA-induced VEGF levels, thereby increasing tumor latency and reducing tumor number.

Assessment of the immunohistochemical data pertaining to the expression of specific markers showed that LU significantly reduced levels of tumor-associated VEGF in all treatment groups $(1,10$, and $25 \mathrm{mg} / \mathrm{kg})$ compared with MPA alone (Fig. 5A). Non-tumor-associated levels of VEGF in mammary glands was significantly reduced by the highest 
Emerged Day 53

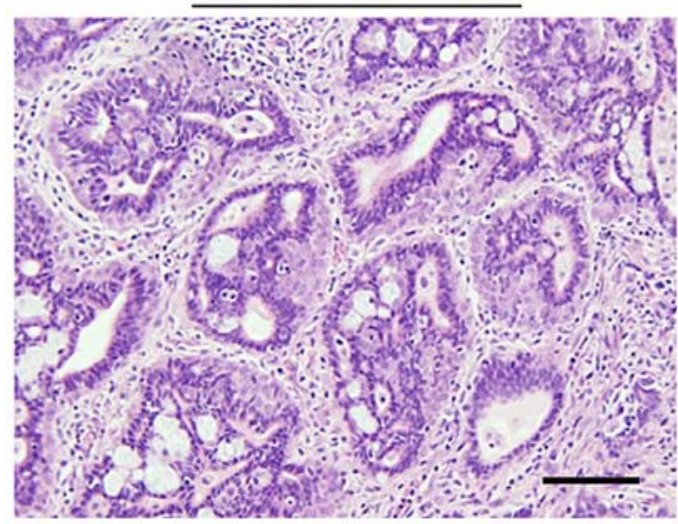

Emerged Day 39

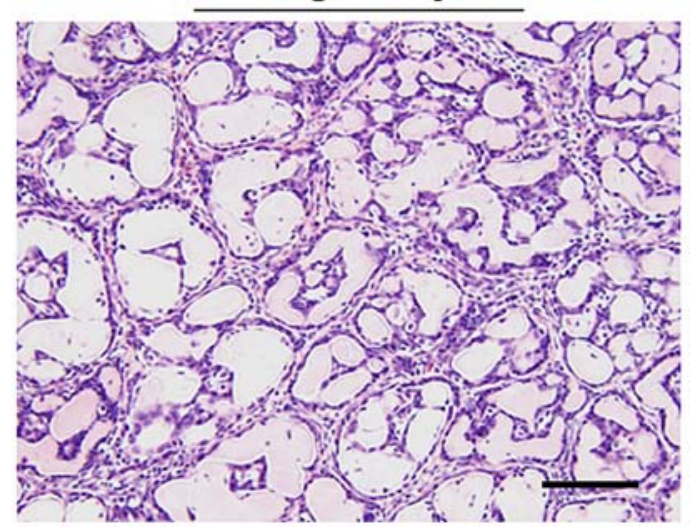

Figure 4. Luteolin induces tumor regression. H\&E staining of MPA-driven LU ( $25 \mathrm{mg} / \mathrm{kg})$ treated tumors. Left, tumor that emerged post-LU treatment (palpated at day 53); this was the only tumor available for analysis from the $25 \mathrm{mg} / \mathrm{kg} \mathrm{LU}$ group shown in Fig. 5. Right, tumor that developed during LU treatment (palpated at day 39), but then regressed. Magnification, x40. Bar represents $100 \mu \mathrm{m}$.

A
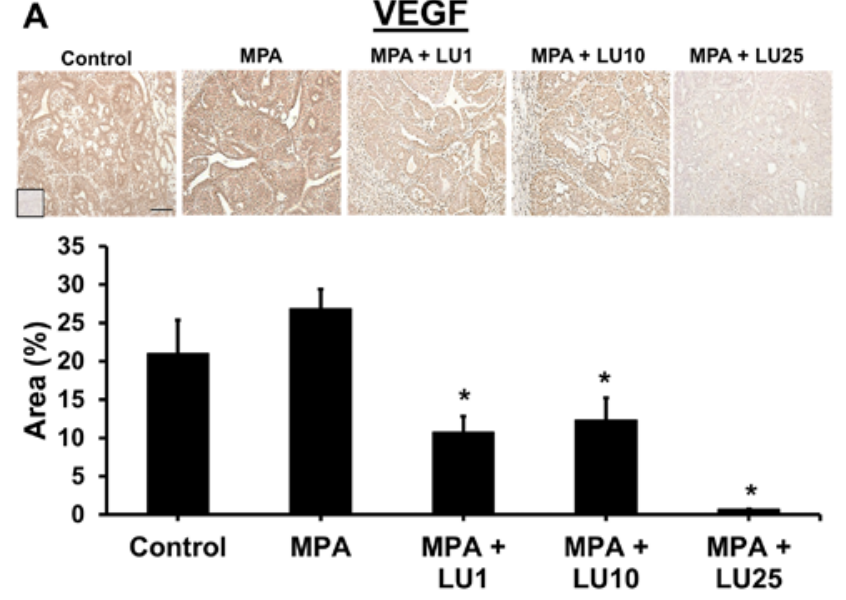

B
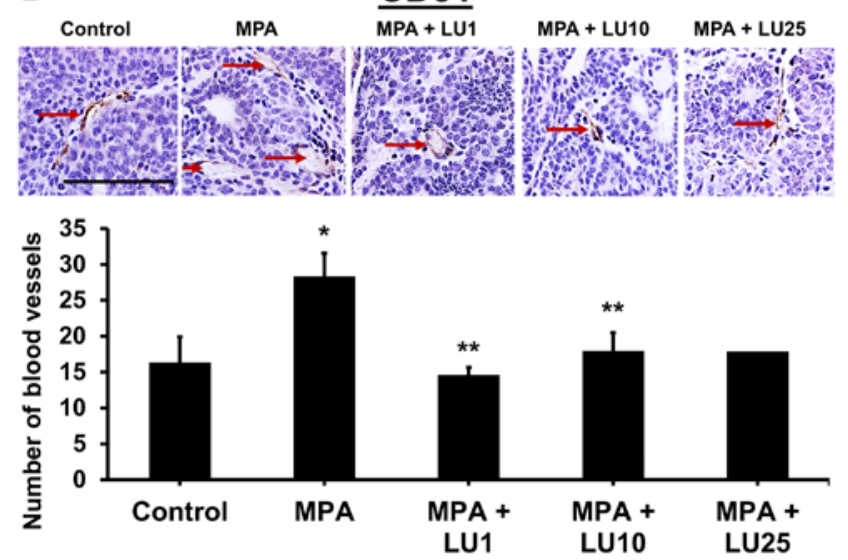

Figure 5. Luteolin reduces expression of VEGF and CD31, markers of angiogenesis, in mammary tumors. Tumors were removed 2 weeks after the last injection of LU (day 59) and tissues subjected to immunohistochemistry Immunohistochemical data from tumors was quantitated per group $(n \geq 3$; except for $25 \mathrm{mg} / \mathrm{kg} \mathrm{LU}$ tumor tissue). (A) Upper panel, representative immunohistochemical analyses of tumor, images were captured at x20 and scale bar represents $100 \mu \mathrm{m}$. VEGF quantification, mean \pm SEM shown in lower panel. VEGF staining in tumor tissues ${ }^{*} \mathrm{P}<0.05$ compared with MPA-treated groups. (B) Upper panel, CD31 staining quantified by number of blood vessels present; representative pictures are at $\mathrm{x} 20$ and scale bar represents $100 \mu \mathrm{m}$. Lower panel, bar graph represents mean \pm SEM number of blood vessels captured at x10 per treatment group, as described in Materials and methods. ${ }^{*} \mathrm{P}<0.05$ compared to control group, while ${ }^{* *} \mathrm{P}<0.05$ compared to MPA-treated group. dose of LU $(25 \mathrm{mg} / \mathrm{kg})$ compared with controls (data not shown). MPA alone had no significant effect on VEGF levels in either tumor or mammary gland tissues though there was a trend towards higher levels of VEGF within tumors derived from MPA treated animals (Fig. 5A and data not shown). Tumors from MPA-treated animals exhibited a higher number of blood vessels compared with controls and all three doses of LU significantly suppressed tumor blood vessel formation (Fig. 5B).

Luteolin increases expression of Ki67, a marker of proliferation, in mammary tumor tissue. Levels of the proliferation marker Ki67 were significantly higher within end-point tumor tissues derived from animals given $10 \mathrm{mg} / \mathrm{kg} \mathrm{LU}$ compared with either MPA alone, MPA $+1 \mathrm{mg} / \mathrm{kg}$ LU or controls (Fig. 6A). Expression of Ki67 was reduced in tumors obtained from the group given $25 \mathrm{mg} / \mathrm{kg} \mathrm{LU}$ compared with either MPA alone or controls, however, this effect was not statistically significant (Fig. 6A). In contrast, compared with controls, levels of Ki67 were significantly higher in non-tumor mammary gland tissue collected from animals given the lowest dose of LU $(1 \mathrm{mg} / \mathrm{kg}$ ) but not in the other treatment groups (data not shown).

TUNEL assays of tumor tissue demonstrated no significant differences in levels of apoptosis between groups treated with LU (Fig. 6B). However, significantly less cell death occurred in non-tumor mammary gland tissue obtained from animals administered $25 \mathrm{mg} / \mathrm{kg} \mathrm{LU}$, compared with controls (data not shown). Taken together, these data suggest that the reduced levels of cell death and increased proliferation observed at the end of the study in some animals given LU may occur in response to the lifting of selective inhibitory pressure on day 46 when LU injections were terminated.

Immunohistochemical analysis of tissue derived from mammary tumors and contra-lateral non-tumor mammary glands showed that in the latter, LU was unable to prevent and/or reverse the formation of hyperplastic lesions arising in response to MPA (please see images in Fig. 5) (data not shown). These data suggest that LU exerts its effects and prevents MPA-induced tumor development in breast tissue at a stage subsequent to the formation of precancerous lesions. 
A

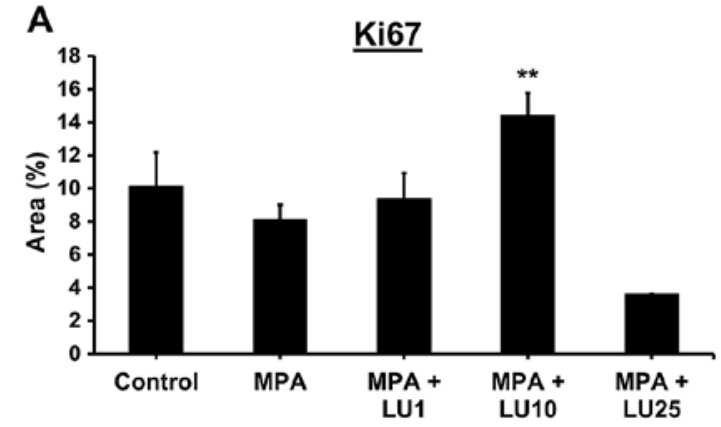

B

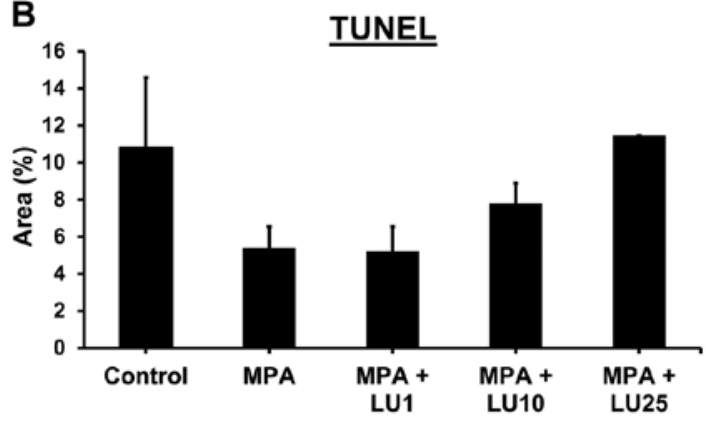

Figure 6. Luteolin increases expression of Ki67, a marker of proliferation, in mammary tumors. Tumors were prepared as previously described in Materials and methods. Immunohistochemistry data from tumors was quantitated per group ( $\mathrm{n} \geq 3$; except for $25 \mathrm{mg} / \mathrm{kg} \mathrm{LU}$ group). (A) Ki67 was quantified and reported as mean \pm SEM. Ki67 staining in tumor tissues. ${ }^{* *} \mathrm{P}<0.05$ compared with control and MPA-treated groups. (B) Tissue was prepared and stained for TUNEL; quantification is reported as mean \pm SEM.

\section{Discussion}

The consumption of combination HRT, which contains both estrogen and progestin, puts millions of postmenopausal women at higher risk of developing breast cancer compared with those taking estrogen alone (2-5). It is therefore imperative that we develop new and effective safe synthetic and/or naturally-occurring compounds with antiprogestin activity that can be taken in conjunction with combination HRT regimens to eliminate the progestin-dependent increase in breast cancer risk. With this in mind, we conducted studies to investigate the ability of the flavonoid LU to act as a preventive compound in MPA-driven breast cancer, given that LU has been shown to have anticarcinogenic properties in other test systems $(22,24)$. Using an established progestin-dependent DMBA mammary tumor model previously developed in our laboratory (17-19), we determined that LU suppresses the development of progestin-driven mammary tumors.

Surprisingly, when LU was used as a chemopreventive agent, a biphasic response was observed, whereby both low and high doses ( 1 and $25 \mathrm{mg} / \mathrm{kg}$ ), but not an intermediate dose $(10 \mathrm{mg} / \mathrm{kg})$, of the flavonoid decreased MPA-induced mammary tumor incidence and increased mammary tumor latency. These observations resulted in a non-monotonic U-shaped dose-response curve (35). It should be noted that treatment with $10 \mathrm{mg} / \mathrm{kg} \mathrm{LU}$, in the absence of MPA, did not cause induction of tumors or toxicity to animals (data not shown), indicating that the flavonoid does not have any inherent tumor-stimulating properties. Importantly, no adverse effects were observed with any of the doses of LU used in these studies.
Having determined that LU is an effective means by which to block the development of progestin-dependent tumors, we sought to elucidate the mechanisms responsible by examining VEGF, blood vessel density and Ki67 expression, as well as conducting TUNEL assays in sections from mammary tumors and contralateral inguinal mammary glands. Because LU treatment stopped two weeks prior to the termination of the study, these results represent the lasting effect of LU on the targeted tissues.

Tumor incidence was low in animals given 1 and $25 \mathrm{mg} / \mathrm{kg}$ $\mathrm{LU}$, an observation which may be explained by a sustained loss of VEGF within tumor tissue resulting in an inability of preneoplastic lesions to form frank tumors, as previously observed with apigenin treatment (19). It is likely that loss of VEGF, which is potently angiogenic, resulted in reduced blood vessel density in regressing tumors, indicating disruption of tumor nourishment. Although LU caused a sustained downregulation of tumor-associated VEGF that was independent of dosage, it had no effect on VEGF levels in non-tumor mammary gland tissue. The ability of LU to significantly reduce both VEGF production and tumor volume in all three dosage groups $(1,10$, and $25 \mathrm{mg} / \mathrm{kg}$ ) is most likely explained by the flavonoid acting as both an antiprogestin and possibly an estrogen/antiestrogen $(23,29,36-38)$. This proposed mechanism could explain why tumor volumes in the $10 \mathrm{mg} / \mathrm{kg}$ group were reduced even though the incidence of tumors in the same group was high. Since LU did not influence progesterone receptor levels (data not shown), its inhibitory effect may be due to its ability to attenuate the post-ligand binding signal transduction pathway normally known to promote VEGF production in tumor cells. For example, hypoxia-inducible factor $\alpha$ (HIF-1 $\alpha$ ) plays an important role in progesterone receptor-mediated VEGF induction (39). Thus, inhibition or downregulation of HIF-1 $\alpha$ may suppress VEGF activity. It is also possible that LU may modify the activity of progesterone receptor post-transcriptionally, as shown by others (40). These possibilities remain to be explored.

Ki67 and TUNEL measurements provided little insight into the mechanism through which LU prevents tumor formation in the MPA-driven DMBA-induced mammary tumor model. While neither Ki67 nor TUNEL signals changed markedly in response to doses of 1 and $25 \mathrm{mg} / \mathrm{kg} \mathrm{LU}$, Ki67 expression was significantly increased in tumor tissue obtained from animals given a dose of $10 \mathrm{mg} / \mathrm{kg} \mathrm{LU}$, suggesting that in this group, circulating levels of LU caused tumor cell proliferation, which in turn resulted in LU losing its ability to control the formation of MPA-driven tumors. Such effects of flavonoids have been reported previously $(19,21)$. It is likely that our inability to gain meaningful data for Ki67 and TUNEL assays is due to the time lag between cessation of LU treatment and tumor collection. During this period when the suppressive pressure of LU was removed, tumor cell proliferation most likely increased, while apoptosis decreased. In future studies we will address this time-lag by collecting tumors at the time $\mathrm{LU}$ treatment is terminated. Suppression of VEGF expression and consequent disruption of angiogenesis by LU may explain why these tumors remained smaller compared with those under the influence of MPA alone, even though tumor incidence was equivalent.

It is well known that progestins increase VEGF in hormone-responsive tumor cells $(12,17,19,41)$. Recent 
evidence suggests that progestins not only provide a microenvironment conducive to growth and metastasis, but that they also enrich the tumor cell population $(42,43)$. It is therefore imperative that we improve the available therapeutic antiprogestin options. The minimal increase in tumor-associated VEGF observed in the MPA group in this study likely occurred as a result of the length of time involved in the experiment. Due to a large number of variously sized (both large and small) tumors present in the first place, maximum tumor growth may have already occurred prior to the end of the experiment, influencing final VEGF levels. Alternatively, seasonal variations may have caused fluctuations in levels of VEGF (44). Nevertheless, it is interesting to note that LU brought about a persistent and significant reduction in VEGF production within mammary tumors, and that suppression of VEGF was independent of dosage. To our knowledge, this is the first study of any lasting effect of LU (i.e., persistent inhibition of VEGF) even when cessation of LU administration occurred at a point well before the end of the experiment. These observations suggest that LU may cause epigenetic changes in the VEGF gene, though this remains to be determined. Importantly, these findings indicate that LU has the ability to prevent tumors from establishing a microenvironment conducive to growth. Our observations also suggest that LU may suppress cancer stem cells since tumor incidence remained low even when LU supplementation was discontinued. Thus in vitro studies to examine the effects of LU on the self-renewal properties of stem-like cells are warranted. Considering that treatment with $25 \mathrm{mg} / \mathrm{kg} \mathrm{LU}$ was nontoxic, resulted in the lowest total number of tumors ( 3 tumors in the $25 \mathrm{mg} / \mathrm{kg}$ group vs. 4 tumors in the $1 \mathrm{mg} /$ $\mathrm{kg}$ group), and largely counteracted the effects of MPA on DMBA-induced mammary cancer by reducing both tumor volume and incidence, we propose that the flavonoid should be further evaluated as a naturally-occurring chemopreventive compound. LU possesses important antitumor properties that may well be extremely advantageous to women who are either undergoing combination HRT or who have already been exposed to this type of therapy. Further studies are justified to elucidate fully the effects of LU in vivo and to gain a better understanding of its potential for human use. In future animal studies, the effects of orally administered LU should be examined, in order to determine the preventive properties of the flavonoid when ingested as a dietary supplement. Such studies would serve as a means of assessing its potential use in humans.

\section{Acknowledgements}

The present study was supported by a peer-reviewed COR award from the University of Missouri College of Veterinary Medicine (Columbia, MO) and by funds from generous donors to the Ellis Fischel Cancer Center, University of Missouri (Columbia, MO). We would like to thank Mr Jason Lee for help with the figures.

\section{References}

1. Siegel R, Ma J, Zou Z and Jemal A: Cancer statistics, 2014. CA Cancer J Clin 64: 9-29, 2014.
2. Chlebowski RT, Hendrix SL, Langer RD, Stefanick ML, Gass M, Lane D, Rodabough RJ, Gilligan MA, Cyr MG, Thomson CA, et al; WHI Investigators: Influence of estrogen plus progestin on breast cancer and mammography in healthy postmenopausal women: The Women's Health Initiative Randomized Trial. JAMA 289: 3243-3253, 2003.

3. Beral V; Million Women Study Collaborators: Breast cancer and hormone-replacement therapy in the Million Women Study. Lancet 362: 419-427, 2003.

4. Rossouw JE, Anderson GL, Prentice RL, LaCroix AZ, Kooperberg C, Stefanick ML, Jackson RD, Beresford SA, Howard BV, Johnson KC, et al; Writing Group for the Women's Health Initiative Investigators: Risks and benefits of estrogen plus progestin in healthy postmenopausal women: Principal results from the Women's Health Initiative randomized controlled trial. JAMA 288: 321-333, 2002.

5. Fahlén M, Fornander T, Johansson H, Johansson U, Rutqvist LE, Wilking $\mathrm{N}$ and von Schoultz E: Hormone replacement therapy after breast cancer: 10 year follow up of the Stockholm randomised trial. Eur J Cancer 49: 52-59, 2013.

6. Horn LC, Schnurrbusch U, Bilek K, Hentschel B and Einenkel J: Risk of progression in complex and atypical endometrial hyperplasia: Clinicopathologic analysis in cases with and without progestogen treatment. Int J Gynecol Cancer 14: 348-353, 2004.

7. Pike MC, Peters RK, Cozen W, Probst-Hensch NM, Felix JC, Wan PC and Mack TM: Estrogen-progestin replacement therapy and endometrial cancer. J Natl Cancer Inst 89: 1110-1116, 1997.

8. Brisken C: Progesterone signalling in breast cancer: A neglected hormone coming into the limelight. Nat Rev Cancer 13: 385-396, 2013.

9. Gonzalez-Suarez E, Jacob AP, Jones J, Miller R, Roudier-Meyer MP, Erwert R, Pinkas J, Branstetter D and Dougall WC: RANK ligand mediates progestin-induced mammary epithelial proliferation and carcinogenesis. Nature 468: 103-107, 2010.

10. Hyder SM, Murthy L and Stancel GM: Progestin regulation of vascular endothelial growth factor in human breast cancer cells. Cancer Res 58: 392-395, 1998.

11. Liang Y, Besch-Williford C, Brekken RA and Hyder SM: Progestin-dependent progression of human breast tumor xenografts: A novel model for evaluating antitumor therapeutics. Cancer Res 67: 9929-9936, 2007.

12. Liang Y, Benakanakere I, Besch-Williford C, Hyder RS, Ellersieck MR and Hyder SM: Synthetic progestins induce growth and metastasis of BT-474 human breast cancer xenografts in nude mice. Menopause 17: 1040-1047, 2010.

13. Liang Y, Wu J, Stancel GM and Hyder SM: p53-dependent inhibition of progestin-induced VEGF expression in human breast cancer cells. J Steroid Biochem Mol Biol 93: 173-182, 2005.

14. Liang Y and Hyder SM: Proliferation of endothelial and tumor epithelial cells by progestin-induced vascular endothelial growth factor from human breast cancer cells: Paracrine and autocrine effects. Endocrinology 146: 3632-3641, 2005.

15. Joshi PA, Jackson HW, Beristain AG, Di Grappa MA, Mote PA, Clarke CL, Stingl J, Waterhouse PD and Khokha R: Progesterone induces adult mammary stem cell expansion. Nature 465: 803-807, 2010.

16. Schramek D, Leibbrandt A, Sigl V, Kenner L, Pospisilik JA, Lee HJ, Hanada R, Joshi PA, Aliprantis A, Glimcher L, et al: Osteoclast differentiation factor RANKL controls development of progestin-driven mammary cancer. Nature 468: 98-102, 2010.

17. Benakanakere I, Besch-Williford C, Schnell J, Brandt S, Ellersieck MR, Molinolo A and Hyder SM: Natural and synthetic progestins accelerate 7,12-dimethylbenz[a]anthracene-initiated mammary tumors and increase angiogenesis in Sprague-Dawley rats. Clin Cancer Res 12: 4062-4071, 2006.

18. Benakanakere I, Besch-Williford C, Carroll CE and Hyder SM: Synthetic progestins differentially promote or prevent 7,12-dimethylbenz(a)anthracene-induced mammary tumors in Sprague-Dawley rats. Cancer Prev Res (Phila) 3: 1157-1167, 2010.

19. MafuvadzeB,BenakanakereI,LópezPérezFR,Besch-WillifordC, Ellersieck MR and Hyder SM: Apigenin prevents development of medroxyprogesterone acetate-accelerated 7,12-dimethylbenz(a) anthracene-induced mammary tumors in Sprague-Dawley rats. Cancer Prev Res (Phila) 4: 1316-1324, 2011.

20. Mafuvadze B, Liang Y, Besch-Williford C, Zhang X and Hyder SM: Apigenin induces apoptosis and blocks growth of medroxyprogesterone acetate-dependent BT-474 xenograft tumors. Horm Cancer 3: 160-171, 2012. 
21. Mafuvadze B, Cook M,Xu Z, Besch-Williford CL and Hyder SM Effects of dietary apigenin on tumor latency, incidence and multiplicity in a medroxyprogesterone acetate-accelerated 7,12-dimethylbenz(a)anthracene-induced breast cancer model Nutr Cancer 65: 1184-1191, 2013.

22. Lin Y, Shi R, Wang X and Shen HM: Luteolin, a flavonoid with potential for cancer prevention and therapy. Curr Cancer Drug Targets 8: 634-646, 2008.

23. López-Lázaro M: Distribution and biological activities of the flavonoid luteolin. Mini Rev Med Chem 9: 31-59, 2009.

24. Seelinger G, Merfort I, Wölfle U and Schempp CM Anti-carcinogenic effects of the flavonoid luteolin. Molecules 13: 2628-2651, 2008.

25. Bagli E, Stefaniotou M, Morbidelli L, Ziche M, Psillas K, Murphy $\mathrm{C}$ and Fotsis T: Luteolin inhibits vascular endothelial growth factor-induced angiogenesis; inhibition of endothelial cell survival and proliferation by targeting phosphatidylinositol 3'-kinase activity. Cancer Res 64: 7936-7946, 2004.

26. Pratheeshkumar P, Son YO, Budhraja A, Wang X, Ding S, Wang L, Hitron A, Lee JC, Kim D, Divya SP, et al: Luteolin inhibits human prostate tumor growth by suppressing vascular endothelial growth factor receptor 2-mediated angiogenesis. PLoS One 7: e52279, 2012.

27. Selvendiran K, Koga H, Ueno T, Yoshida T, Maeyama M, Torimura T, Yano H, Kojiro M and Sata M: Luteolin promotes degradation in signal transducer and activator of transcription 3 in human hepatoma cells: An implication for the antitumor potential of flavonoids. Cancer Res 66: 4826-4834, 2006

28. Luo H, Jiang BH, King SM and Chen YC: Inhibition of cell growth and VEGF expression in ovarian cancer cells by flavonoids. Nutr Cancer 60: 800-809, 2008

29. Nordeen SK, Bona BJ, Jones DN, Lambert JR and Jackson TA: Endocrine disrupting activities of the flavonoid nutraceuticals luteolin and quercetin. Horm Cancer 4: 293-300, 2013.

30. Fang J, Zhou Q, Shi XL and Jiang BH: Luteolin inhibits insulin-like growth factor 1 receptor signaling in prostate cancer cells. Carcinogenesis 28: 713-723, 2007.

31. Zhou Q, Yan B, Hu X, Li XB, Zhang J and Fang J: Luteolin inhibits invasion of prostate cancer PC3 cells through E-cadherin. Mol Cancer Ther 8: 1684-1691, 2009.

32. Samy RP, Gopalakrishnakone P and Ignacimuthu S: Anti-tumor promoting potential of luteolin against 7,12-dimethylbenz(a) anthracene-induced mammary tumors in rats. Chem Biol Interact 164: 1-14, 2006.

33. Faustino-Rocha A, Oliveira PA, Pinho-Oliveira J, Teixeira-Guedes C, Soares-Maia R, da Costa RG, Colaço B, Pires MJ, Colaço J, Ferreira R, et al: Estimation of rat mammary tumor volume using caliper and ultrasonography measurements. Lab Anim (NY) 42: 217-224, 2013.
34. Tomayko MM and Reynolds CP: Determination of subcutaneous tumor size in athymic (nude) mice. Cancer Chemother Pharmacol 24: 148-154, 1989.

35. Vandenberg LN, Colborn T, Hayes TB, Heindel JJ, Jacobs DR Jr, Lee DH, Shioda T, Soto AM, vom Saal FS, Welshons WV, et al: Hormones and endocrine-disrupting chemicals: Low-dose effects and nonmonotonic dose responses. Endocr Rev 33: 378-455, 2012

36. Markaverich BM, Shoulars K and Rodriguez MA: Luteolin regulation of estrogen signaling and cell cycle pathway genes in MCF-7 human breast cancer cells. Int J Biomed Sci 7: 101-111, 2011.

37. Le Bail JC, Varnat F, Nicolas JC and Habrioux G: Estrogenic and antiproliferative activities on MCF-7 human breast cancer cells by flavonoids. Cancer Lett 130: 209-216, 1998.

38. Zand RSR, Jenkins DJ and Diamandis EP: Steroid hormone activity of flavonoids and related compounds. Breast Cancer Res Treat 62: 35-49, 2000

39. Carroll CE, Liang Y, Benakanakere I, Besch-Williford C and Hyder SM: The anticancer agent YC-1 suppresses progestin-stimulated VEGF in breast cancer cells and arrests breast tumor development. Int J Oncol 42: 179-187, 2013.

40. Knutson TP and Lange CA: Tracking progesterone receptor-mediated actions in breast cancer. Pharmacol Ther 142: 114-125, 2014.

41. Hyder SM, Chiappetta C and Stancel GM: Pharmacological and endogenous progestins induce vascular endothelial growth factor expression in human breast cancer cells. Int J Cancer 92: 469-473, 2001

42. Cittelly DM, Finlay-Schultz J, Howe EN, Spoelstra NS, Axlund SD, Hendricks P, Jacobsen BM, Sartorius CA and Richer JK: Progestin suppression of miR-29 potentiates dedifferentiation of breast cancer cells via KLF4. Oncogene 32: 2555-2564, 2013

43. Horwitz KB, Dye WW, Harrell JC, Kabos P and Sartorius CA: Rare steroid receptor-negative basal-like tumorigenic cells in luminal subtype human breast cancer xenografts. Proc Natl Acad Sci USA 105: 5774-5779, 2008.

44. Löscher W, Mevissen M and Häussler B: Seasonal influence on 7,12-dimethylbenz[a]anthracene-induced mammary carcinogenesis in Sprague-Dawley rats under controlled laboratory conditions. Pharmacol Toxicol 81: 265-270, 1997. 